\title{
Dual Axes Solar Tracker
}

\author{
Ahmad Imran bin Ibrahim ${ }^{1}$, Farah Diyana binti Abdul Rahman ${ }^{2}$, Muazzin bin Rohaizat ${ }^{3}$ \\ ${ }^{1,3}$ Departement of Mechatronics Engineering, International Islamic University Malaysia, Malaysia \\ ${ }^{2}$ Department of Electrical and Computer Engineering, International Islamic University Malaysia, Malaysia
}

\begin{tabular}{l}
\hline \hline Article Info \\
\hline Article history: \\
Received Aug 20, 2017 \\
Revised Dec 5, 2017 \\
Accepted Jan 20, 2018 \\
\hline
\end{tabular}

Keyword:

Dual axes

Light dependent resistor

Photovoltaic

Solar tracker

\begin{abstract}
Photovoltaic (PV) is one of the most important sources of renewable energy in the world. Its current efficiency could be increased up to $60 \%$ by using dual axes solar tracker, which maximise PV exposure to sun. The most important component in dual axes solar tracker is sensing location of the sun. Four light dependent resistors (LDR) are used as the sensors, connected to potentiometers to increase their accuracy. Arduino UNO is used as the controller to control two stepper motors. Two experiments have been carried out, where the tolerance of the LDR has been found to be $0.05 \mathrm{~V}$ and the calibration of the four LDRs to have the error of $0.03 \mathrm{~V}$. Both experiments proved the capability of LDR for dual axes solar tracker and potentiometer to increase their accuracy.
\end{abstract}

Copyright $(0) 2018$ Institute of Advanced Engineering and Science.

All rights reserved.

\section{Corresponding Author:}

Ahmad Imran bin Ibrahim,

Department of Mechatronics Engineering,

International Islamic University Malaysia,

P.O. Box 10, 50728 Kuala Lumpur, Malaysia.

Email: imran@iium.edu.my

\section{INTRODUCTION}

Photovoltaic (PV) is one of the most promising renewable energy in the world [1], [2]. The popularity of the PV is due to its capability for a small scale installation. However, the efficiency of existing PV is only about $11-19 \%$ and the best commercial PV is about $23 \%$, while the best research cell is up to $30 \%$ [3]. Efficiency of the PV could be maximised by facing the PV perpendicular to sunlight, which could increase power output between $30 \%$ to $60 \%$ compared to stationary PV [4].

Therefore, mechanised PV has potential in increasing the output without sacrificing its footprint. There are two types of mechanised PV; single axis and dual axes. Single axis mechanised PV normally allows motion from sunrise to sunset [5]. It is suitable for countries along the equator where the sun positions do not differ much during summer and winter. The advantage of the single axis is it only requires a single actuator which is easier to control and has less weight. However, it requires a proper installation that allows the mechanism to follow sun movements.

On the other hand, dual axes mechanised PV is more flexible due to its ability to follow the sun in any directions regardless of the orientation of the initial installation of the PV [6]. An extra actuator is the only trade-off for gaining its flexibility and adaptability of the system, which makes it possible to install in any moving objects without losing sunlight. It is a more efficient method compared to single axis [7]

\section{RESEARCH METHOD}

Dual axes solar tracker consists of two main systems; mechanical system and electrical system. Figure 1 shows the mechanical system where most of the small parts have been 3D printed, while the large parts are constructed using Perspex. Perspex is the chosen material due to its lightweight property and easy to cut when compared to steel or aluminium. The mechanical system comprises of two set of gear train that 
move the PV into azimuth and altitude direction, as well as the holder for PV and its sensors. The electrical system could be divided into three subsystems; solar sensing, control, and actuator. Solar sensing is the most critical part, where position of the sun is established by using proper sensor. The signal from the sensor regarding the sun position is transmitted to controller to actuate the PV to a perpendicular position to the sun. The overall electrical system connection between these items is shown in Figure 2. It could be observed in the figure that four potentiometers have been connected in series with four LDRs. The usage of the potentiometer is to calibrate the reading from the LDRs.
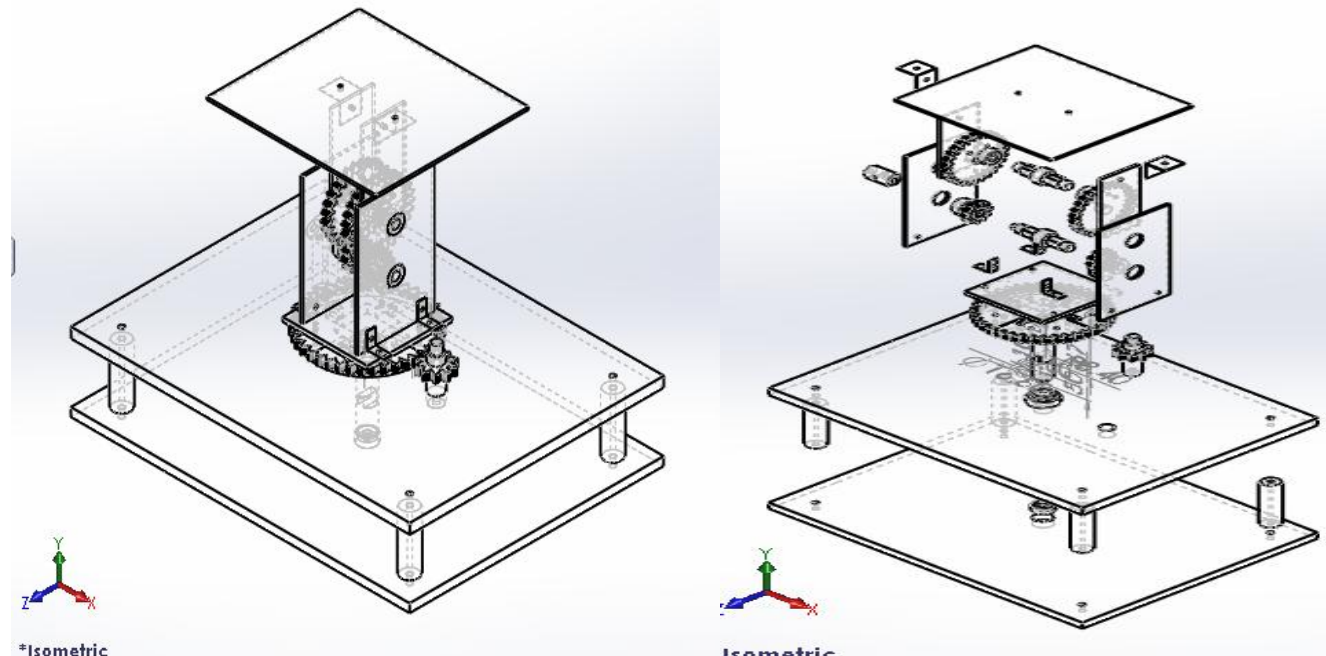

Figure 1. Overall mechanical system of dual axes solar tracker, on the left is the assembled view and on the right is the explode view of the system

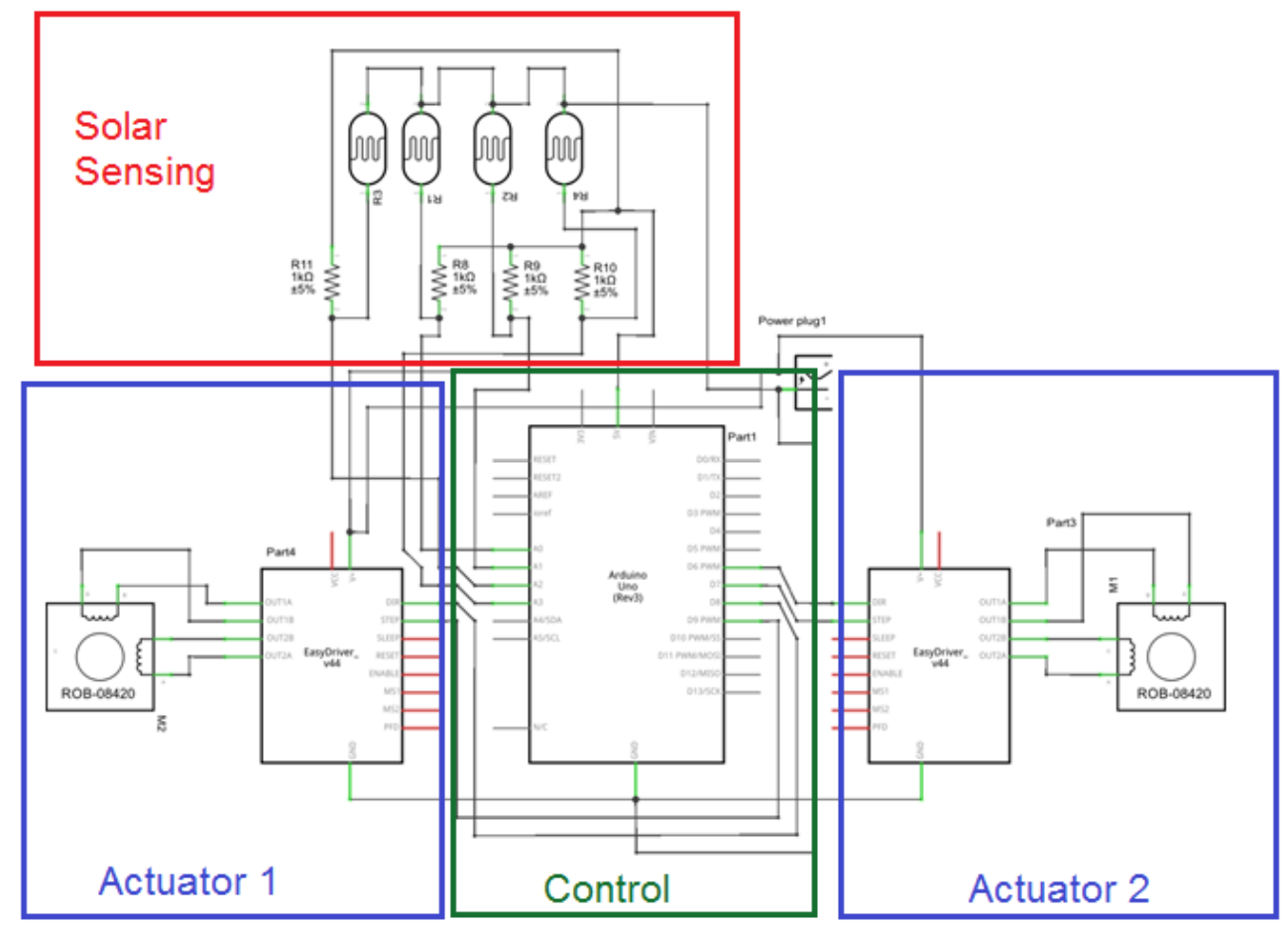

Figure 2. Schematic diagram for electrical parts of dual axes solar tracker which consists of solar sensing, control and two actuators 
The first subsystem consists of four light dependent resistors (LDR) as sensors to determine position of the sun with respect to the PV. LDR is chosen due to its low cost and accessibility. Furthermore, it is a simpler system as compared to stainless steel canister design by Holambe et. al. [8]. Four LDRs are located side by side such as edges of a square of about $3 \mathrm{~mm}$ apart. This design is similar to the one proposed by Sohaq et. al [9] when they combined the LDRs and the camera with image processing method for accurate and efficient system. However, the integration of image processing system could only increases the performance of PV further $1.45 \%$ to $20 \%$ of LDR system with the complexity of image processing and extra computing power.

The control subsystem used Arduino Uno as the controller as it has enough pins to connect to all four LDRs which require analog inputs. The controller also has enough pulse width modulation (PWM) output pins to support two electrical motors. These two electrical motors are required for two steps process to orient PV to sunlight direction; azimuth and altitude as shown in Figure 3. The first two LDR sensor readings are compared to determine azimuth direction of the sun while the other two are then compared to decide altitude position of the sun.

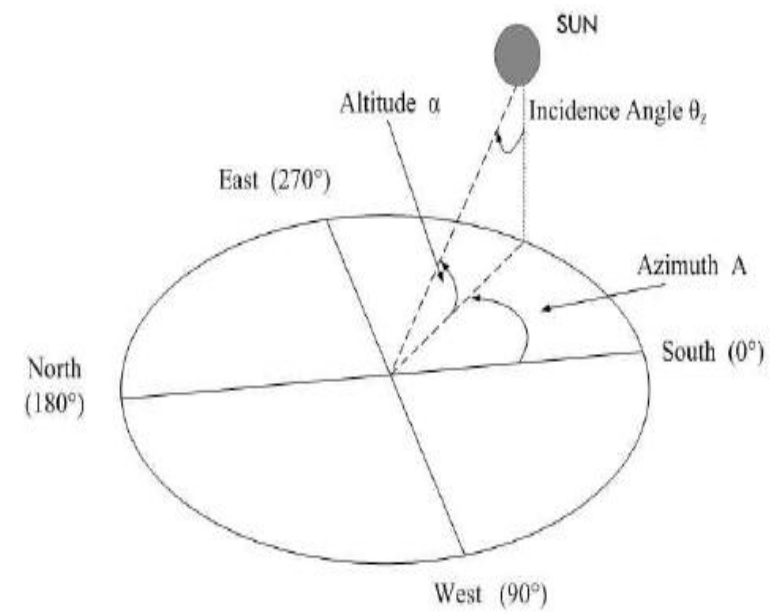

Figure 3. Azimuth and altitude orientation that use to determine position of the sun [10]

There are two actuators required to actuate the PV into azimuth and altitude direction of the sun for the actuator subsystem. Two stepper motor s are used to actuate azimuth and altitude position of the PV. Stepper motor is chosen due to its high torque capability as compared to DC motor.

There are researches on passive tracker to orient PV towards the sun which can be found in [8], [11]. Their systems are using stainless steel canisters filled with compressed gas fluid with low boiling point and gravity to actuate position of the PV towards the sun. The system does not require any sensor, controller and actuator to operate. However, this system is very sensitive to wind, which could misalign its position deeming it impracticality.

\section{RESULTS AND ANALYSIS}

Each component of the dual axes solar tracker has been tested to identify the suitable setup for the controller. Two experiments have been carried out to determine the relationship between single LDR reading and the angle of light, and calibration of four LDR readings to each other with regards to perpendicular light source.

\subsection{LDR reading relation to light source angle}

An experiment has been carried out to observe the reading for LDR with different angle of light towards LDR. The angle of light from the LDR is taken from 0 degree until 180 degrees, where the result of the experiment is as shown in Figure 4. It could be observed in the graph that the LDR gives a reading of $0.46 \mathrm{~V}$ when light is 90 degrees to the LDR which is the determination point for position of the sun when it is perpendicular to the PV. However, the voltage reading for $60,70,80$, and 100 degrees are all $0.52 \mathrm{~V}$ which shows $0.06 \mathrm{~V}$ difference compared to 90 degrees reading. Therefore, the tolerance for the voltage reading should be lower than $0.05 \mathrm{~V}$ to make sure that the PV is perpendicular to the sun. 


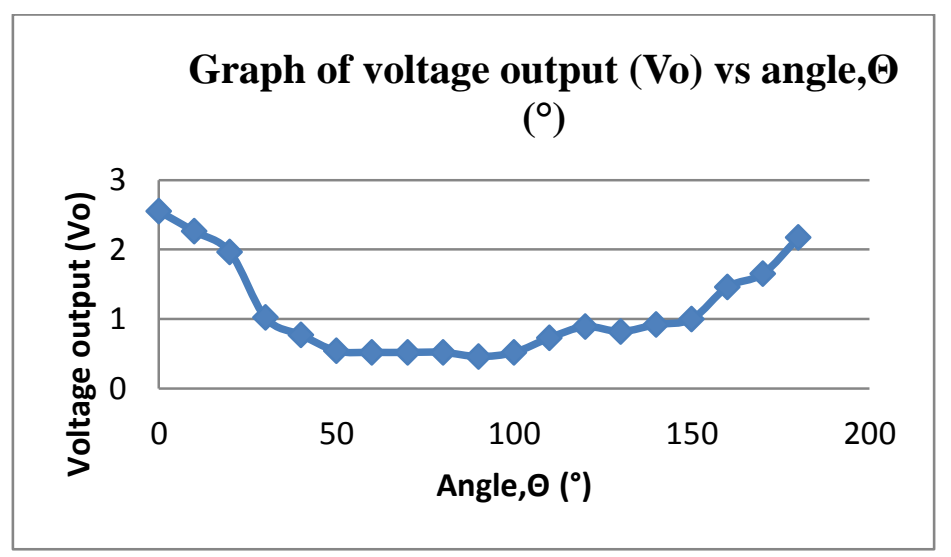

Figure 4. Result of outputs from LDR vs. angle of light towards LDR

\subsection{Calibration of four LDR readings}

The ideal situation is when the PV is perpendicular to the sun, indicated when all four LDRs are having the same voltage reading. However, in reality, all four LDRs will have slightly different readings to one another. These different readings should not be higher than $0.05 \mathrm{~V}$ because that is the tolerance limit for the LDR to be used in the controller. Therefore, a potentiometer is connected in series with each LDR to make sure their readings are the same when the source of light is perpendicular to the PV. Another purpose of the potentiometer is to shift the sensitivity of the LDR towards the sunlight region. Figure 5 shows the digital readings for the four LDRs before calibration. It could be observed that all LDRs give different values with the same amount of light.

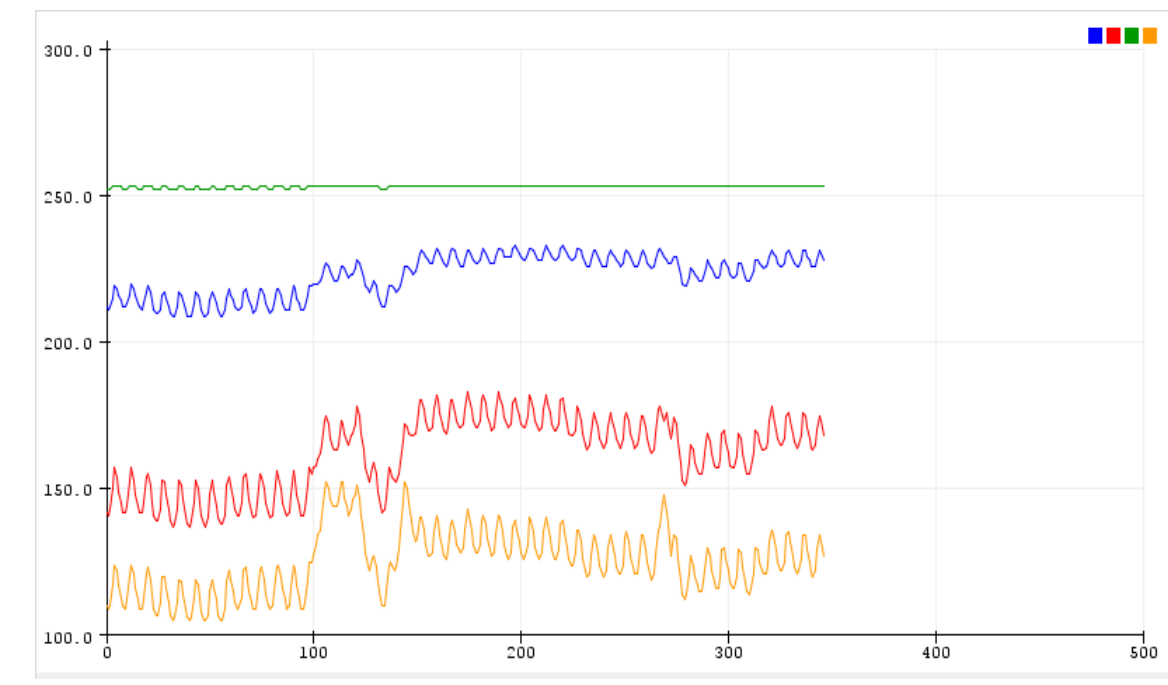

Figure 5. Readings of the four LDRs before calibration process, Y-axis represents the voltage reading in centivolt, while $\mathrm{X}$-axis represents the time in millisecond

Each of the potentiometer is adjusted so that all the LDR readings are the same with the same amount of light as shown in Figure 6. It could be observed in the graph, there are some light disturbance at the beginning of the reading. However, there are some error readings between LDRs after calibration which is about $0.01 \mathrm{~V}-0.03 \mathrm{~V}$. This error is an acceptable value because it is below the tolerance value of $0.05 \mathrm{~V}$.

A prototype of dual axes solar tracker has been developed as shown in Figure 7, based on the results established. This prototype is developed to test the capability of the LDR detecting sunlight and aligning it toward the sun. It could be integrated with the PV to compare its performance using LDR, with and without potentiometer. 


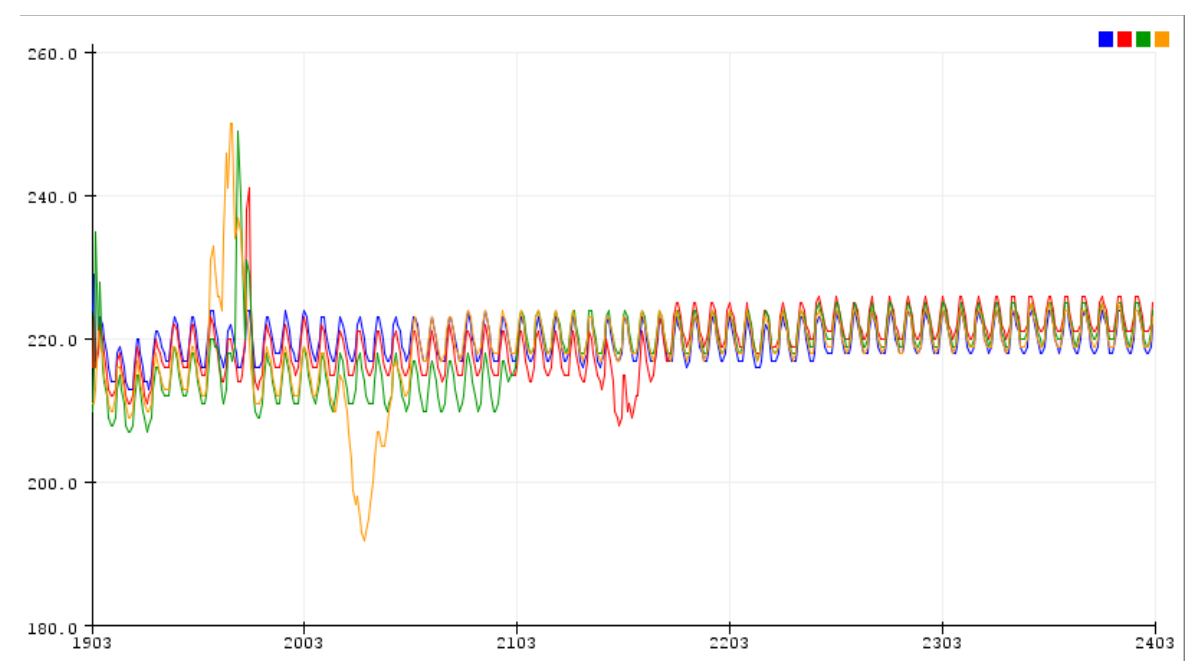

Figure 6. Readings of the four LDRs after calibration process, Y-axis represents the voltage reading in centivolt, while $\mathrm{X}$-axis represents the time in millisecond

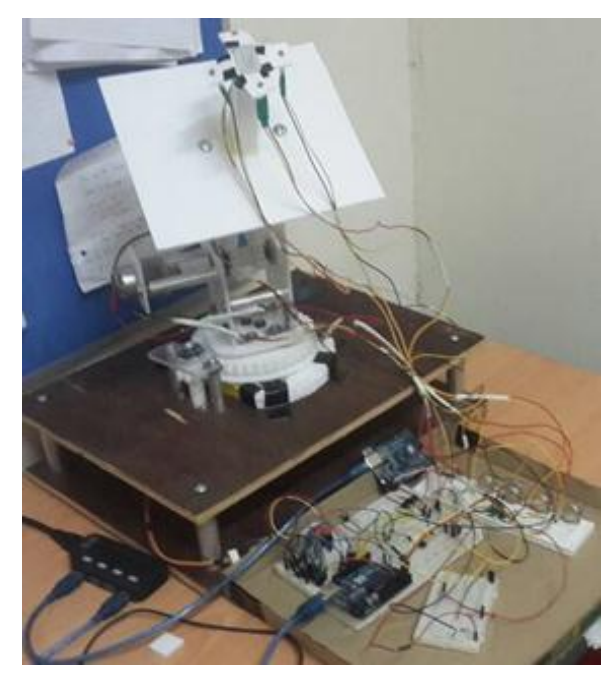

Figure 7. Prototype of dual axes solar panel

\section{CONCLUSION}

Dual axes solar tracker could increase the efficiency of the PV up to $60 \%$ as compared to static PV. However, further improvement of the performance of dual axes solar PV depends on the capability of the system to align with the sunlight. From the experiment, it has been determined that the difference between totally aligned and 30 degrees angle off is just $0.05 \mathrm{~V}$ from the LDR reading. There is an effort using image processing to further improve dual axes PV performance. However, the cost of the integrated image processing is very significant if compare to $20 \%$ of the performance increase. This research is focusing on developing better LDR sensor which could increase the PV performance of tracking sunlight. This research managed to develop dual axes solar tracker for the PV by using Arduino UNO, LDRs and stepper motors. Potentiometer is used in series with each LDR to increase the efficiency of the LDR because the tolerance of the reading for it to be exactly perpendicular to sunlight is $0.05 \mathrm{~V}$.

\section{ACKNOWLEDGEMENTS}

The authors acknowledge the financial assistance of this research which is supported by the Research Initiative Grant Scheme (RIGS) with the grant number RIGS16-348-0512 and International Islamic University Malaysia (IIUM). 


\section{REFERENCES}

[1] Carrasco, J.M., et al., "Power-electronic systems for the grid integration of renewable energy sources: A survey", IEEE Transactions on industrial electronics, 2006, vol. 53, no. 4, pp. 1002-1016.

[2] Krautmann, J., \& Zhu, J, 2012, "Photovoltaic Solar Energy Systems: Market Trends in the United States", International Journal of Applied Power Engineering (IJAPE), vol. 1, no. 3, pp. 123-128.

[3] Archer, M. D., M. A. Green, "Clean electricity from photovoltaics", vol. 4, 2014, World Scientific.

[4] Khan, M. T. A., et al., "Design and construction of an automatic solar tracking system", in Electrical and Computer Engineering (ICECE), 2010, International Conference on. 2010.

[5] Das, S., Sadhu, P., Pal, N., \& Mukherjee, S, 2014, "Single Axis Automatic Solar Tracking System Using Microcontroller", Indonesian Journal of Electrical Engineering and Computer Science, vol. 12, no. 12, pp. 8028-8032.

[6] Singh, P., Pahuja, R., Karwasra, M., Beniwal, S., Bansal, M., \& Dadhich, A, 2016, "Dual Axis Solar Tracking System for Solar Panel", Bulletin of Electrical Engineering and Informatics, vol. 5, no. 4, pp. 403-411.

[7] Zhang, X., et al., "Two tracking control method to improve solar cell photoelectric efficiency", in Control and Decision Conference (CCDC), 2016 Chinese.

[8] Holambe, P.R., D. Talange, and V. Bhole, "Motorless solar tracking system", in Energy Systems and Applications, 2015 International Conference on. 2015.

[9] Sohag, H.A., et al., "An accurate and efficient solar tracking system using image processing and LDR sensor", in Electrical Information and Communication Technology (EICT), 2015 2nd International Conference on. 2015.

[10] Zhan, T.-S., et al., "Design and Implementation of the Dual-axis Solar Tracking System", in Computer Software and Applications Conference (COMPSAC), 2013 IEEE 37th Annual, 2013, IEEE.

[11] Parmar, N., A.N. Parmar, and V.S. Gautam, "Passive solar tracking system", Int J Emerg Technol Adv Eng, 2015, vol. 5 , no. 1 , pp. 138-145.

\section{BIOGRAPHIES OF AUTHORS}
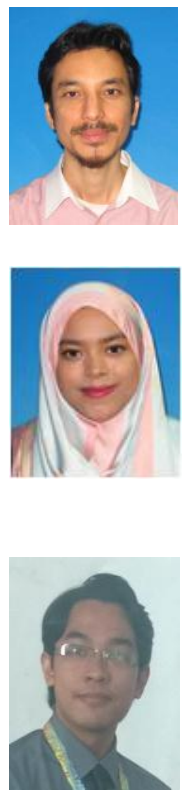

Ahmad Imran bin Ibrahim obtained his PhD from Mechanical Department, University of Bristol, UK in 2016. He has been appointed as Assistant Professor in Mechatronics Department, Faculty of Engineering, International Islamic University Malaysia (IIUM). Prior to that, he was a Senior Executive -Engineering in Sime Darby Technology Centre, overlook research projects for Sime Darby Plantation. His research interests include biomechanical design, optimised robotic design and bioinspired robot. He is a member of IEEE and registered in Board of Engineers Malaysia (BEM).

Farah Diyana Abdul Rahman obtained her PhD degree from Department of Electrical and Electronic Engineering, University of Bristol, UK in 2015. She has been appointed as an Assistant Professor in Electrical and Computer Engineering Department, Faculty of Engineering, International Islamic University Malaysia (IIUM). Her research interest includes image and video processing, video quality evaluation, multimedia transmission and wireless communication systems. She is an active member of the IEEE, a registered member of the Board of Engineers Malaysia (BEM) and Institute of Engineers Malaysia (IEM).

Muazzin Rohaizat is a student from Mechatronics Department, Faculty of Engineering, International Islamic University Malaysia (IIUM). He started his study in IIUM in 2013 and will be graduating in 2018. His final year project contributes towards this article. 